\title{
Premisas psicológicas para la formación en investigación de los estudiantes de pregrado
}

\author{
Psychological premises for research training of undergraduate students
}

\author{
Rosa Ordoñez V. ${ }^{1}$, Margarita León G. \\ Universidad Laica Vicente Rocafuerte de Guayaquil, Ecuador \\ (RECIBIDO 22-10 2014, AcEPTAdo 02-12-2014)
}

\begin{abstract}
RESUMEN
La investigación formativa es un proceso integral, consecutivo, reflexivo, sistemático, controlado y crítico, que permite descubrir nuevos conocimientos en cualquier campo del desarrollo humano, lo que requiere mucho esfuerzo intelectual de ideas y razonamientos que permitan transformar una realidad. La presentación de este tema tiene como propósito plantear alternativas pedagógicas sobre sustento psicológico, para mejorar las habilidades de investigación, tanto de los docentes como de los estudiantes, conociendo primero la realidad actual de los procesos de investigación formativa, para, luego, replantear estrategias de conocimientos técnicos pedagógicos para realizar investigaciones en etapa de formación de pregrado, estableciendo los términos idóneos para investigar, lo que implica dominio de técnicas para analizar, comprender y aplicar la información científica dirigida a la solución de problemas de la realidad. La investigación científica exige el desarrollo de habilidades y modos de actuación, dirigidos a interactuar positiva y creadoramente con la naturaleza y la sociedad, de manera responsable. Las premisas psicológicas de la investigación formativa centran su atención en el papel que les corresponde a las habilidades investigativas y su desarrollo, a partir de los criterios de frecuencia, periodicidad, flexibilidad y complejidad, solo alcanzables en la relación de la teoría y la práctica.
\end{abstract}

Palabras claves: Investigación científica, investigación formativa, habilidades investigativas.

1 Maestría en Educación Superior, Universidad de Guayaquil, con estudios doctorales actuales en la UNMSM Lima-Perú. 


\begin{abstract}
The formative research is an integral process, sequenced, reflexive, systematic, controlled, and critical. Allowing it to discover new knowledge in any field of human development, which requires much intellectual effort to create ideas and reasoning that enable to transform the reality. The presentation of this topic has aims to propose pedagogical alternatives on the basis of their livelihood psychological, in order to improve the research skills of professor and students. It is important first to know the current reality of the formative research process to implement strategies of teaching technical skills to conduct investigations during undergraduate studies, establishing suitable terms to investigate which implies a mastery of techniques to analyze, understand, and apply scientific information directed to the solutions of problems of the world. Scientific research demands the development of skills and modes of action, aimed to interact positively and creatively with nature and society, in a responsible manner. The psychological premises of the formative research focused on the role that correspond to the investigative skills and their development based on the criteria of frequency, periodicity, flexibility, and complexity only attainable in a relationship of theory and practice.
\end{abstract}

Keywords: Scientific research, formative research, research skills.

\title{
CONFERENCIA
}

\section{CONTEXTO}

El proceso de enseñanza-aprendizaje universitario está dirigido a personas en la edad juvenil, las cuales son personalidades en pleno desarrollo; por tanto, hay que partir de ellas para educarlas y, a la vez, el resultado de este proceso es contribuir a su mejora continua y de calidad. Para ello, la personalidad es el punto de partida y el fin de la educación, por lo que es atinado aceptar este principio como fundamental en la psicología de la educación.

\section{PERO ¿QUÉ ES LA INVESTIGACIÓN FORMATIVA?}

Investigación es un procedimiento reflexivo, sistemático, controlado y crítico que permite descubrir nuevos hechos o datos, relaciones o leyes, en cualquier campo del conocimiento humano. Proceso sistemático y metodológico de indagación y estudio que se sigue para incrementar el conocimiento. Es un trabajo intelectual que se realiza con el fin de generar nuevos conocimientos y, en consecuencia, hacer aportaciones valiosas a la humanidad (Ortiz, 2013:126).

La investigación requiere del esfuerzo intelectual del investigador para llevar a la comprobación, en la práctica, de las ideas y razonamientos que tiene a partir de esta propia práctica. El conocimiento entonces se genera en la práctica y regresa a ella para transformarla.

Toda investigación parte de una idea, que constituye el primer acercamiento a la realidad que se pretende estudiar. La mayoría de estas ideas iniciales son vagas, imprecisas y será necesario analizarlas cuidadosamente, para lograr poco a poco, peldaño a peldaño, transformarlas en planteamientos estructurados, y la vía para lograrlo es el diseño del proyecto de la investigación (Borrego, 2009:5). 
La investigación se desarrolla mediante un proceso y parte de la identificación de un área temática en la cual se desea dar respuesta a un problema. Para tal fin se diseña o planifica un proyecto de investigación, que comienza por plantear de manera precisa y concisa el problema y de soportarlo en términos de justificación, objetivos, propósito, y marco teórico e hipótesis (Borda, 2013:16).

Los conocimientos en general y el proceso de aprendizaje del ser humano, varían y se actualizan con rapidez, como respuesta a los avances científicos y tecnológicos, fruto de la aplicación de la investigación científica, que busca dar respuesta a los problemas que día a día deben enfrentar los profesionales en las áreas del saber en las que se desempeñan (Borda, 2013:1).

Saber cómo realizar una investigación, así como reportar sus resultados, es muy importante para el desempeño de la vida académica, profesional y laboral; da la pauta para desarrollar trabajos con los elementos de validez científica y metodológica. Desafortunadamente se arrastran vicios, falta de aptitudes y conocimiento de sus fundamentos metodológicos, y de la manera de reportar sistemáticamente sus resultados (Lara, 2011).

Partiendo de esta apreciación de Lara, es que las autoras razonan que existen falencias no solamente de los estudiantes, sino, aún más, de los docentes, lo que implica que recientemente se está trabajando en conocimientos técnicos pedagógicos de cómo realizar investigaciones en etapa de formación de pregrado. De hecho es necesario aclarar los términos consultar investigar. Lo más común es escuchar en el aula de clases, a la gran mayoría de los docentes, decir realicen una investigación, cuando se trata de consultas bibliográfica de un determinado tema. No tener bien definido esos conceptos elementales para llevar a cabo una investigación como tal ocasiona la continuidad de expresar conceptos erróneos, que conducen a la confusión y desinterés por parte de los involucrados.

La discontinuidad en los procesos de capacitación para formación en investigación, dirigida primero a los docentes, que son responsables de llevar a cabo una verdadera línea de investigación áulica, así como los proyectos de investigación para la presentación de tesis, conlleva agudizar aún más la debilidad en la formación de los estudiantes para la investigación. Esta discontinuidad de capacitación implica que no existe el mismo lenguaje entre los docentes cuando se habla de investigación. Cada profesor tiene una metodología de investigación diferente, que dispersa los escasos conocimientos de pocos estudiantes en cuanto a desarrollo de investigaciones formativas dentro del proceso curricular.

La forma en que se planifican las investigaciones a nivel formativo con los estudiantes de pregrado es poco adecuada, pues no hay un sistema de planeamiento investigativo para que los docentes se guíen y direccionen sus investigaciones, de manera sistemática, ordenada, planificada, bien estructurada y con un lenguaje universal. Dentro de la unidad académica, por lo menos, se trabaja a nivel individual en cuanto a investigaciones dirigidas para los estudiantes de pregrado. 
Esta problemática se agudiza aún más cuando existe cierta resistencia por parte de algunos docentes para la aplicación de métodos estadísticos en la investigación, ya que aún no se conoce cuáles son los métodos estadísticos más utilizados por el claustro docente. No se han creado grupos de investigación de docentes de la universidad que se reúnan regularmente para discutir, socializar, recrear y compartir temas de investigación áulica, que fomenten la neguentropía investigativa y alimenten nuevas ideas investigativas con mayor o menor grado de complejidad.

Al realizar una observación directa de campo, y gracias a los años en el entorno educativo, se puede determinar que el gran problema es la deficiente cultura para la lectura y la investigación, que dificulta el desarrollo de los procesos de investigación en la universidad ecuatoriana, no solo desde la óptica docente, sino, aún más, de parte de los estudiantes. La plataforma de una buena investigación es el conocimiento de la línea base y el marco teórico, y para ello es necesario concentrar los esfuerzos en una lectura comprensiva, ya que no solo se trata de observar el objeto, sino de encontrar los aspectos sobresalientes de la investigación a través de la lectura de otras investigaciones concernientes a realidades del mismo tema. Y esto lo destaca el diario el universo, del 20 de Julio de este año, en la que manifiesta que "parte de la deficiencia educativa es que el estudiante ecuatoriano no lee, y le cuesta cuando se le exige. El Informe 2012 del Centro Regional para el Fomento del Libro en América Latina y el Caribe de la Unesco señala que en el Ecuador la cifra de lectura es de 0.5 libros al año por persona. En Colombia, de 4.1 libros; Argentina 4.6; España, 11.1 libro.

En general, se presenta un escaso conocimiento de la metodología y las diferentes teorías de la investigación, así como, de los procedimientos o pasos para realizar las investigaciones formativas y de titulación, concretamente. Este escaso conocimiento surge de la mediana capacitación continua para dirigir proyectos de investigación formativa o áulica y aquellos proyectos de investigación para las futuras titulaciones.

Las asignaturas relacionadas a la investigación están en el programa, pero a partir del cuarto año de estudios, cuando deberían de estar desde el primer semestre para que los estudiantes ya tengan nociones de la investigación, o que la investigación esté implícita en cada asignatura, independientemente del área de estudio o especialidad. Los estudiantes tienen una idea distorsionada de lo que es investigación; de tal manera que, hasta concluir la carrera, no conocen la metodología para investigar cuando se trata de su proyecto de titulación. Esto muchas veces ocasiona malestar entre el docente tutor y el estudiante, ya que en muchas ocasiones el estudiante cree que el docente debe darle todo hecho, sin que se esfuerce en leer la información, saber parafrasear y peor aún, hacer un resumen.

No existe un perfil idóneo de docentes que se dedique como especialización a la investigación, sea esta de tipo formativa o áulica y de titulación, ya que el docente para investigar, y más aún para enseñar a los estudiantes a investigar, debe tener no solamente actitud, sino también aptitud. Los estudiantes carecen de la motivación interna y externa, necesaria para involucrase en un proceso de 
investigación, de tal manera que le huyen a la práctica investigativa, ya que no existe un proceso de investigación formativa para que el estudiante se involucre en el deseo de formarse como investigadores.

Cuando se envía una investigación no se establece el objetivo de ella por lo tanto no permite enfocar la investigación. La práctica común de los estudiantes, cuando se les envía una consulta bibliográfica, es hacer un pobre análisis y en muchas ocasiones se limitan a copiar y pegar la información, desconociendo muchas veces incluso la fuente de la consulta, y muchas veces con la aceptación de los docentes. Y esto se hace notorio por el desconocimiento en planteamientos y temas de investigación, cuando se trata de proyectos de investigación de titulación.

Las investigaciones no generan el efecto multiplicador del conocimiento para nuevos problemas de investigación, y por ende, las investigaciones que se generan en el proceso quedan rezagadas en el camino.

No hay un semillero de investigadores, ni de docentes ni de estudiantes, ya que no existe un proceso de investigación formativa; primero se "investiga" y luego se forma, lo que ocasiona confusión y pérdida de tiempo. Esto es, porque no existe un proceso de investigación formativa y que permita reconocerse como una necesidad para la universidad y el entorno social. Se investiga por cumplir un requisito académico, como cumplimiento de carga horaria de investigación o de titulación, y no se la involucra como una necesidad para la docencia, para la universidad y finalmente para la sociedad.

\section{PROPÓSITO DE ESTUDIO}

La discusión de este tema tiene como propósito plantear alternativas, incluyendo aspectos psicológicos, para mejorar las habilidades de investigación, tanto de los docentes como de los estudiantes, conociendo primero la realidad actual de los procesos de investigación formativa, para luego, replantear estrategias de conocimientos técnicos pedagógicos para realizar investigaciones en etapa de formación de pregrado, para estudiantes y docentes, estableciendo los términos idóneos para investigar, así como la diferencia entre investigar y consultar un determinado tema.

Para ello es necesario establecer una serie de acciones, como definir bien esos conceptos elementales para llevar a cabo una investigación para no crear confusión y buscar el interés de investigar con los elementos de juicio por parte de los involucrados en los procesos de investigación formativa.

Determinar y establecer procesos de capacitación para la investigación formativa dirigida a los docentes para una verdadera línea de investigación áulica, para que el lenguaje entre los docentes y los estudiantes involucrados en la investigación, sea eficiente y el más adecuado.

Establecer parámetros de planificación para una investigación áulica que permita realizar investigaciones a nivel formativo con los estudiantes de pregrado de manera óptima. 
Regular un sistema de planeamiento investigativo para que los docentes se guíen y direccionen sus investigaciones de manera sistemática, ordenada, planificada, bien estructurada y con un lenguaje universal dentro de la unidad académica, de esta manera se trabajará a nivel multidisciplinario en cuanto a investigaciones áulicas.

Formar redes internas de investigación de docentes de la universidad, que se reúnan regularmente para discutir, socializar, recrear, compartir temas de investigación, que alimente nuevas ideas de investigación, cada vez con mayor grado de complejidad. Esta agrupación tendría como característica principal incentivar la cultura de la lectura y la investigación para mejorar el desarrollo de los procesos de investigación en la universidad, no solo desde la visión docente sino, también, por parte de los estudiantes, ya que la base de una investigación es la lectura de diferentes fuentes bibliográficas, para sustentar la línea base y el marco teórico.

Establecer la metodología y los procedimientos o pasos para realizar las investigaciones formativas y de titulación, con capacitación continua para dirigir proyectos de investigación formativa o áulica y aquellos proyectos de investigación para las futuras titulaciones, involucrando cada vez más a los estudiantes, de tal manera que se sientan motivados a formarse como investigadores.

Generar el efecto multiplicador del conocimiento para nuevos problemas de investigación, formando semilleros de investigadores, tanto docentes como estudiantes, para los procesos de investigación formativa, que permita reconocer la necesidad de la universidad de investigar e investigar, no como un requisito académico, sino como necesidad imperiosa desde la docencia hasta los estudiantes, y que signifique un impacto para la sociedad.

En la investigación científica en la que participan los profesionales en formación, se debe ir creando una cultura científica que sea la vía esencial para la transformación del entorno, con la que todos están responsabilizados y comprometidos. Por ello, es necesario desarrollar la cultura científica en las instituciones educativas, como un factor importante en el proceso formativo.

Se coincide con la conclusión de Chirino Ramos (2010), en que la cultura científica es una rama de la cultura que implica dominio de información para analizar, comprender y aplicar la información científica dirigida a la solución de problemas contenidos en la demanda social. Ella incluye habilidades, sentimientos, modos de actuación, dirigidos a interactuar positiva y creadoramente con la naturaleza y la sociedad, de manera responsable.

El desarrollo de las habilidades científico investigativas tiene un sustento básico desde el punto de vista psicológico.

Las habilidades son un componente importante de la actividad científica. Leontiev (1981) y seguidores desarrollaron importantes estudios acerca de la estructura de las habilidades. La habilidad es entendida como dominio de una acción que permite regular su ejecución de forma consciente y con la flexibilidad que demanda la situación o contexto en que se ejecuta; está, desde el punto de vista estructural, 
constituida por las operaciones que la conforman, lo que se corresponde con su función ejecutora en la actividad del sujeto.

Cruz Tejeda y otros (2011) hacen un análisis interesante al respecto. Estos autores plantean que la relación operación-habilidad (acción) es una relación dialéctica, pues las operaciones de una habilidad pueden constituir, en otras condiciones, habilidades en sí mismas. Para que una acción devenga habilidad, su ejecución debe ser sometida a frecuencia, periodicidad, flexibilidad y complejidad.

La frecuencia se relaciona con el número de repeticiones necesarias para que la acción se refuerce, se consolide y se desarrolle como habilidad. Varía no solo en dependencia de su complejidad, sino también se debe considerar el nivel de desarrollo del sujeto que la ejecuta, por lo que su planificación debe basarse en el diagnóstico inicial de los estudiantes y, a partir al establecer estrategias diferenciadas para los estudiantes.

La periodicidad plantea la necesidad de retomar cada cierto tiempo la habilidad, para que no se olvide, y su planificación está también en dependencia del nivel de desarrollo alcanzado por los estudiantes. Para que la acción se transforme en habilidad, debe ser ejecutada en diversas tareas, con diferentes conocimientos, en diferentes condiciones (flexibilidad). Esto se justifica por la relación dialéctica conocimiento-habilidad. Esta flexibilidad debe ir acompañada de un aumento progresivo en la complejidad de las tareas que se asignan a lo largo de la carrera.

Dentro de las cualidades que denotan que la acción se ha materializado en habilidad, están: mayor grado de generalización, sistematización, síntesis de sus operaciones, independencia en la ejecución, éxito en los resultados.

Si se desea lograr el objetivo planteado en el perfil del profesional, que se dirige a la formación de un profesional capaz de enfrentar científicamente los retos de las condiciones cambiantes del mundo laboral, que aporte soluciones a los problemas del mismo, adelantándose al futuro con proyectos transformadores, entonces, las acciones generalizadoras del método científico deben ser sometidas a ejecución frecuente, periódica, flexible y con complejidad ascendente, de forma gradual, como premisas para que puedan devenir habilidades científico investigativas, al evidenciar el modo en que el estudiante se relaciona con el objeto de estudio a partir de la lógica del conocimiento científico.

Las habilidades científico investigativas son entendidas, por tanto, como el “... dominio de las acciones generalizadoras del método científico que potencian al individuo para la problematización, teorización y comprobación de su realidad profesional, lo que contribuye a su transformación sobre bases científicas" (Cruz Tejeda, y otros, 2011. P. 27).

El desarrollo de las habilidades científico-investigativas durante la formación de los profesionales (investigación formativa) se sustenta en los principios y conceptos fundamentales del enfoque histórico cultural desarrollado por L.S. Vigotsky 
(1896-1934). Los principios de la naturaleza social de la psiquis humana y del determinismo dialéctico e histórico del desarrollo psíquico, como guías para la comprensión, orientación y dirección de la investigación formativa resultan esenciales.

Se parte de considerar que el ser humano es social por naturaleza, por lo que se forma y se desarrolla en la actividad y en la comunicación que establece con el medio en que vive y está en constante cambio y transformación, aunque mantiene una estabilidad relativa en su personalidad.

En la unidad de la actividad y la comunicación, los estudiantes pueden alcanzar un desarrollo de sus potencialidades y modificar las características que poseen en cada momento de su desarrollo psicológico, a partir de la relación con los otros y con los objetos de su entorno, mediante un proceso de internalización o interiorización de lo interpsíquico en intrapsíquico.

Los procesos de cambio ocurren en su interacción con lo que les rodea, en una relación de interdependencia, en la que cada uno va logrando niveles cada vez más altos de autonomía y autodeterminación, a partir de las ayudas que solicita y recibe de los demás, en situaciones de intersubjetividad (León García y otros, 2013).

Esto se expresa en la ley genética general del desarrollo cultural planteada por Vigotsky (1987): “... cualquier función en el desarrollo cultural del niño aparece en escena dos veces, en dos planos: primero como algo social, después como algo psicológico; primero entre la gente, como una categoría interpsíquica, después, dentro del niño, como una categoría intrapsíquica” p. 161).

Se asume, por lo tanto, la concepción vigotskiana acerca de la relación entre educación y desarrollo, o sea que la educación conduce y guía el desarrollo, va por delante a partir de tener en cuenta el desarrollo alcanzado y las potencialidades del sujeto, para plantearle exigencias crecientes que le permitan avanzar, con las ayudas pertinentes, hacia niveles superiores de desarrollo.

Se parte también del estudio de la situación social del desarrollo como expresión de la interrelación entre lo interno y lo externo, y las interacciones con los distintos contextos educativos que influyen en el desarrollo de los estudiantes, a partir del diagnóstico del nivel de desarrollo alcanzado y de la zona de desarrollo próximo

Se concuerda plenamente con Vigotsky (1987) cuando define la zona de desarrollo Próximo como: “... la distancia entre el nivel real de desarrollo, determinado por la capacidad de resolver independientemente un problema, y el nivel de desarrollo potencial, determinado a través de la resolución de un problema bajo la guía de un adulto o en colaboración con otro compañero más capaz" (Vigotsky, 1987, p. 37).

Se explica la formación investigativa del estudiante universitario como resultado de la interrelación entre sus condiciones internas: desarrollo alcanzado (nivel de desarrollo actual) y potencial (zona de desarrollo próximo); y las condiciones externas: condiciones sociales de vida y educación (influencias de los educadores y de los contextos educativos ya mencionados). 
En la investigación formativa tiene un papel fundamental la mediación, otro concepto aportado por el enfoque histórico cultural. La mediación instrumental y la mediación social resultan esenciales para comprender los mecanismos del desarrollo humano, al permitir entender el papel de la actividad y el de la comunicación, en especial la importancia de la interrelación del estudiante en formación con el docente como la persona que puede brindar las ayudas que necesita para enfrentar las tareas, los problemas y los conflictos relacionados con la actividad investigativa.

La mediación permite al educador conocer, además del nivel de desarrollo alcanzado, las potencialidades que el estudiante posee. Las ayudas se brindan en el mismo momento en que se realiza el diagnóstico.

La investigación formativa es un proceso de aprendizaje. La comprensión que tiene el enfoque histórico cultural del aprendizaje como proceso de apropiación de la cultura es fundamental para lograr una adecuada formación de los estudiantes. Significa que estos hacen suya la experiencia histórico social concretada en los productos materiales (objetos) y espirituales (arte, ciencia, tecnología, costumbres, entre otros) que ha aportado la humanidad; en un proceso activo y creativo, en el cual, con la ayuda del educador o de otro compañero más capaz, construye los contenidos investigativos e incorpora nuevos elementos a sus conocimientos, habilidades y hábitos, a su experiencia individual y a su personalidad; o enriquece y modifica los que ya poseía.

Constituyen también fundamentos psicológicos de la investigación formativa el concepto de sentido personal y la categoría actividad planteados por Leontiev (1981). El sentido personal expresa la significación que para el sujeto adquiere lo que le rodea y que determina su implicación en los hechos y eventos de la realidad; y la categoría actividad explica la manifestación estructural y funcional de la interacción del sujeto con los objetos, lo que permite su estudio y transformación.

En la misma línea del enfoque histórico-cultural, lo planteado por Davidov (1979, 1988) también sustenta la investigación formativa. Este autor propone el enfoque deductivo en el proceso de enseñanza-aprendizaje como parte de los procesos formativos, lo que implica partir de la esencia y para establecer su presencia en los casos particulares que se necesitan para apropiarse de ella, lo que requiere enseñar solo lo fundamental (Davidov, 1988).

La investigación formativa en la universidad debe verse como un proceso de cooperación entre los estudiantes y los docentes, en condiciones que faciliten la apropiación de los contenidos científicos. Bermúdez Morris y Pérez Martín, (2010) señalan que la cooperación de “... acciones conjuntas que implican la realización armónica de las tareas, la colaboración en la toma de decisiones y la responsabilidad compartida en función del logro de los objetivos comunes." (p.10).

Nunca se debe olvidar que los jóvenes que ingresan a la universidad han tenido todo un desarrollo psicológico y educativo precedente, como resultado de las influencias de su familia, de los niveles de enseñanza anteriores y de su historia personal, por lo que cada educando debe ser caracterizado de manera peculiar y 
única, sin aspirar nunca a la homogeneización, porque el ser humano es heterogéneo por naturaleza. Mientras más diferentes sean los estudiantes, mejor desarrollan su personalidad. Siempre la caracterización psicológica de la edad juvenil tendrá un papel orientador con fines formativos, pero nunca con un carácter normativo o de igualitarismo (Ortiz, 2006).

Los estudiantes universitarios se encuentran en las etapas juvenil y adulta, por lo que resulta indispensable conocer las características más generales de los jóvenes y adultos para poder influir en su formación profesional.

\section{IMPORTANCIA DEL PAPEL DE LOS DOCENTES EN LA INVESTIGACIÓN FORMATIVA}

La única pretensión de que la enseñanza y la investigación constituyan una unidad tiene como antecedente el convencimiento de que un docente-investigador es capaz de generar nuevas y mejores condiciones sociales (Rico, 2002).

Desde luego que no faltan los críticos de esta apreciación, y el argumento al que con mayor frecuencia se recurre es el que la formación institucionalizada de docentesinvestigadores propiciaría solamente una especie de intelectualización excesiva que, tarde o temprano, hará que el docente descuide su práctica. (Rico, 2002). Es como considerar que se pierde un gran docente y se gana un mal investigador.

Por ello, resulta de gran importancia la investigación de la práctica del propio docente, es decir que una acción presupone necesariamente a la otra, pues sin la práctica docente propia no existiría el objeto de investigación específico (Rico, 2002). Este aspecto constituye una gran diferencia en cuanto a los estilos y características de la investigación educativa. La propuesta consiste en elevar el nivel de calidad de la educación (Rico, 2002).

El autor citado con anterioridad propone estrategias con respecto a las actitudes del docente, del director y/o de los demás compañeros de trabajo frente a la investigación:

- Mostrarse entusiasta y convincente.

- Instituir foros de discusión mientras dure el proyecto.

- Acordar criterios de validez, considerando la opinión de los demás.

- Relacionarse con otros centros de trabajo.

- Manipulación positiva.

- Avanzar en el trabajo, aun sin la "bendición" del director.

- Toma de decisiones colegiadas, etc.

A pesar de todas las dificultades que entraña lo anteriormente expuesto, en nuestra realidad no es imposible que un docente típico pueda investigar su propia práctica 
y aun el currículo formal, ya sea recibiendo cursos dentro de un salón de clases o investigando con alguien que posea mayor experiencia en el campo investigativo.

El proceso de la investigación y la formación de investigadores es, pues, una situación que reúne muchos ángulos que la tornan compleja.

El maestro puede adquirir las destrezas pedagógicas espontáneamente en la propia práctica cotidiana y no hay necesidad de sistematizar su apropiación. La subestimación que hace esta posición de la formación teórica científica pedagógica la hace extensiva también a la investigación educacional, por lo que desconoce totalmente la importancia de que el maestro domine la metodología de la investigación y mucho menos que la aplique de manera sistemática a la solución de los problemas educativos con los que se enfrenta cotidianamente.

\section{REFERENCIAS BIBLIOGRÁFICAS}

Bermúdez Morris, R. y L. M. Pérez Martín (2010). Modelo de los procesos formativos en el primer año del Curso Regular Diurno de las especialidades técnicas de la Licenciatura en Educación. (Resultado de proyecto de investigación). UCPETP. La Habana (en soporte digital).

Borda, Mariela (2013). El proceso de investigación: visión general de su desarrollo. Editorial Universidad del Norte, Colombia.

Borrego, Maricel (2009). Dossier de metodología de la investigación. $1^{\text {a }}$. Edición. El Cid Editor. Argentina.

Chirino Ramos, M.V. (2010). La formación inicial investigativa en la Universidad de Ciencias Pedagógicas. UCPEJV: La Habana (material en soporte digital).

Cruz Tejeda, N. y otros (2011). La formación de los profesionales de la educación ante los retos de la educación superior contemporánea. Sello Editor Educación Cubana. La Habana.

Davidov, V.V. (1979). Tipos de generalización en la enseñanza. Editorial Pueblo y Educación, Ciudad de La Habana.

La enseñanza escolar y el desarrollo psíquico. Editorial Progreso, Moscú, 1988.

González Rey, F. (1995). Comunicación, Personalidad y Desarrollo. Editorial Pueblo y Educación, Ciudad de La Habana.

Lara, Erica (2011). Fundamentos de investigación: un enfoque por competencias. Editorial Alfaomega. México.

León García, M. y otros (2013). Fundamentos y referentes teórico-metodológicos que sustentan la formación de la fuerza laboral de nivel medio en Cuba. (Resultado de proyecto de investigación). UCPETP. La Habana (en soporte digital).

Leontiev, A.N (1981). Actividad, Conciencia y Personalidad. Editorial Pueblo y Educación, Ciudad de La Habana. 
Ortiz Uribe Frida Gisela (2013). Diccionario de metodología de investigación científica. $3^{\mathrm{a}}$. Edición, México: Limusa.

Ortiz, Emilio (2006). Fundamentos psicológicos del proceso educativo universitario. Universidad De Holguín Oscar Lucero Moya (Uholm).

Pérez Rodríguez, G. (2005). Fundamentos que deben regir la formación investigativa de los maestros. Propuesta teórica desde el constructivismo socio-histórico cultural. ISPEJV. La Habana.

Rico, Pablo (2002). Vademécum del educador (Teoría e Investigación) Universidad Pedagógica Nacional, Zitácuaro, Michoacán, México.

Rojas, R. (1995). Formación de investigadores educativos. Una propuesta de investigación, Plaza y Valdés Editores, México, pp. 30-31.

Vigotsky, L. S. (1987). Historia del desarrollo de las funciones psíquicas superiores. Editorial Científico Técnica, La Habana.

Walker, R. (1989). Métodos de investigación para el profesorado, Ediciones Morata, S. A., Madrid, pp. 219-222. 